\title{
Group-theoretical approach to the calculation of quantum work distribution
}

\author{
Zhaoyu Fei ${ }^{1}$ and H. T. Quan ${ }^{1,2, *}$ \\ ${ }^{1}$ School of Physics, Peking University, Beijing 100871, China \\ ${ }^{2}$ Collaborative Innovation Center of Quantum Matter, Beijing 100871, China
}

(Received 5 August 2019; published 13 December 2019)

\begin{abstract}
Usually the calculation of work distributions in an arbitrary nonequilibrium process in a quantum system, especially in a quantum many-body system, is extremely cumbersome. For all quantum systems described by quadratic Hamiltonians, we propose a universal method for solving the work distribution of quantum systems in an arbitrary driving process by utilizing the group-representation theory. This method enables us to efficiently calculate work distributions where previous methods fail. In some specific models, such as the time-dependent harmonic oscillator, the dynamical Casimir effect, and the transverse XY model, the exact and analytical solutions of work distributions in an arbitrary nonequilibrium process are obtained. Our work initiates the study of quantum stochastic thermodynamics based on group-representation theory.
\end{abstract}

DOI: 10.1103/PhysRevResearch.1.033175

\section{INTRODUCTION}

In the past two decades, a great breakthrough in the field of nonequilibrium thermodynamics is the discovery of fluctuation theorems [1] and the establishment of stochastic thermodynamics [2,3]. It extends the usual definition of work, heat, and entropy production in a thermodynamic process from ensemble-averaged quantities to trajectory-dependent quantities. Based on these extensions, the second law is sharpened and rewritten into equalities (fluctuation theorems) for arbitrary nonequilibrium processes. For an isolated quantum system, a proper definition of the trajectory work is defined through the so-called two-point measurement [4,5], which preserves the non-negativity of the probabilities of trajectories and Jarzynski equality at the same time [6,7]. As is known, the work distribution of a system provides a great deal of information about a nonequilibrium process [8-10], which is an analog to the partition function encoding essential information about an equilibrium state. However, very few analytical results of work distributions of quantum systems have been obtained so far. In the literature, the few exceptions are associated with either specific models (e.g., a forced harmonic oscillator [11-13], a harmonic oscillator with a time-dependent frequency [14,15], quenched Luttinger liquid at zero temperature [8], a diving scalar field [16], or a transverse Ising model at zero temperature [15]) or very special protocols (e.g., the sudden quench protocol [17-23] or the quantum adiabatic protocol [24,25]). Hence, to develop a universal method to efficiently calculate the work distribution

\footnotetext{
*htquan@pku.edu.cn

Published by the American Physical Society under the terms of the Creative Commons Attribution 4.0 International license. Further distribution of this work must maintain attribution to the author(s) and the published article's title, journal citation, and DOI.
}

for various quantum systems in an arbitrary nonequilibrium process is one of the most challenging problems in this field.

In this article, we propose a universal method which is based on the Lie-group theory to calculate the work distribution for quantum systems described by quadratic Hamiltonians [26,27]. Such Hamiltonians are ubiquitous in physics. Examples include the harmonic oscillator, some lattice models, phonons and Bloch electrons in solids, identical particles in an external potential, some models in quantum field theory [16], and many other physical models within the mean-field approximation, e.g., superconductors and superfluids [27]. Our work provides efficient methods to obtain solutions for arbitrary work protocols. And in many cases, the analytical solution can be obtained explicitly, which not only has pedagogical value, but also brings important insights into the design of optimal protocols for thermal machines [28]. Moreover, our method reduces the cost of computational resources from the exponential growth [29] to the polynomial growth with the number of the modes. Since the work distribution provides a great deal of information about thermodynamics in a nonequilibrium process, our method will hopefully significantly advance the development of quantum stochastic thermodynamics, especially for quantum many-body systems.

\section{TWO-POINT MEASUREMENT SCHEME}

In this scheme, the trajectory work for an initial canonical ensemble is determined by the projective measurements over the Hamiltonians before and after the work protocol. Suppose the system evolves from time $t=t_{0}$ to time $t=t_{1}$ with the initial state $\hat{\rho}$ and the time-dependent Hamiltonian $\hat{H}(t)$. For simplicity, let $\hat{\rho}$ commute with $\hat{H}\left(t_{0}\right)$. We measure the energy of the system at the initial and final time over the Hamiltonians $\hat{H}_{0}=\hat{H}\left(t_{0}\right)$ and $\hat{H}_{1}=\hat{H}\left(t_{1}\right)$ respectively. Also, we assume the states of the system are projected into $\left|E_{m}^{0}\right\rangle$ and $\left|E_{n}^{1}\right\rangle$ after the corresponding measurements. Here, $\left\{\left|E_{m}^{0}\right\rangle\right\}$ and $\left\{\left|E_{n}^{1}\right\rangle\right\}$ are the instantaneous orthonormal bases, i.e., $\hat{H}_{0}\left|E_{m}^{0}\right\rangle=E_{m}^{0}\left|E_{m}^{0}\right\rangle$ and $\hat{H}_{1}\left|E_{n}^{1}\right\rangle=E_{n}^{1}\left|E_{n}^{1}\right\rangle$. Then the trajectory work for the above 
realization is given by $w_{n m}=E_{n}^{1}-E_{m}^{0}$. After repeating the above procedure with the same canonical ensemble and the same driving protocol numerous times, the work distribution is obtained as follows:

$$
P(w)=\sum_{m, n}\left\langle E_{m}^{0}|\hat{\rho}| E_{m}^{0}\right\rangle\left|\left\langle E_{n}^{1}|\hat{U}(t)| E_{m}^{0}\right\rangle\right|^{2} \delta\left(w-w_{n m}\right),
$$

where $\hat{U}\left(t_{1}, t_{0}\right)=\mathcal{T} \exp \left[-i \int_{t_{0}}^{t_{1}} d s \hat{H}(s)\right]$ is the unitary evolution operator (we set $\hbar=1$ in this letter) and $\mathcal{T}$ is the timeordered operator. For theoretical analysis, it is a better choice to consider the Fourier transform of the work distribution which is known as the characteristic function of work (CFW) $G(v)[30]$

$$
G(v)=\int_{t_{0}}^{t_{1}} d w P(w) e^{i v w}=\operatorname{Tr}\left(e^{i v \hat{H}_{1}^{H}} e^{-i v \hat{H}_{0}} \hat{\rho}\right),
$$

where the index $H$ in $\hat{H}_{1}^{H}$ indicates that the operator is in the Heisenberg picture, i.e., $\hat{O}^{H}(t)=\hat{U}^{\dagger}\left(t, t_{0}\right) \hat{O}(t) \hat{U}\left(t, t_{0}\right)$. It is worth mentioning that $G(v)$ encodes the same information as $P(w)$.

\section{CALCULATING METHOD OF THE CFW}

As long as the Hamiltonian is a quadratic polynomial of bosonic or fermionic operators and the initial state is a thermal equilibrium state, the $\mathrm{CFW}$ is solvable. In the following, we elucidate our method for arbitrary work protocols.

For later convenience, we first introduce some notation. In a Hilbert space, let $\hat{a}_{i}\left(\hat{b}_{i}\right)$ and $\hat{a}_{i}^{\dagger}\left(\hat{b}_{i}^{\dagger}\right), i=1,2, \ldots, n$, denote the annihilation and creation operators for bosons (fermions). These operators can be considered as the elements of two column vectors, $\boldsymbol{\alpha}=\left(\hat{a}_{1}, \hat{a}_{2}, \ldots, \hat{a}_{n}, \hat{a}_{1}^{\dagger}, \hat{a}_{2}^{\dagger}, \ldots, \hat{a}_{n}^{\dagger}\right)^{T}$ and $\boldsymbol{\beta}=\left(\hat{b}_{1}, \hat{b}_{2}, \ldots, \hat{b}_{n}, \hat{b}_{1}^{\dagger}, \hat{b}_{2}^{\dagger}, \ldots, \hat{b}_{n}^{\dagger}\right)^{T}$ [31], where $T$ denotes the matrix transpose.

Now, without losing generality, we first consider a timedependent bosonic system whose Hamiltonian can be written as

$$
\hat{H}(t)=\frac{1}{2} \boldsymbol{\alpha}^{T} A(t) \boldsymbol{\alpha}+B^{T}(t) \boldsymbol{\alpha}+C(t),
$$

where $A(t)$ is a $2 n \times 2 n$ matrix, $B(t)$ is a $2 n$-dimensional column vector, and $C(t)$ is a scalar. Here, $A(t), B(t)$, and $C(t)$ are work parameters that are controlled by an external agent. From Eq. (2), we see that the calculation of the CFW can be decomposed into two steps. The first step is the calculation of $\hat{H}_{1}^{H}$, and the second step is to calculate the trace of the product of several exponential operators. Based on these facts, the CFW for systems described by the Hamiltonian [Eq. (3)] can be obtained according to the following procedure:

(1) Solve the time dependence of $\boldsymbol{\alpha}^{H}(t)$. Let $\boldsymbol{\alpha}^{H}(t)=$ $\left(\hat{a}_{1}^{H}(t), \hat{a}_{2}^{H}(t), \ldots, \hat{a}_{n}^{H}(t), \hat{a}_{1}^{\dagger H}(t), \hat{a}_{2}^{\dagger H}(t), \ldots, \hat{a}_{n}^{\dagger H}(t)\right)^{T}$ denote $\boldsymbol{\alpha}$ in the Heisenberg picture. Then $\boldsymbol{\alpha}^{H}(t)$ satisfies the following equation:

$$
\frac{d}{d t} \hat{\alpha}_{j}^{H}(t)=-i\left[\hat{\alpha}_{j}^{H}(t), \hat{H}^{H}(t)\right] \quad j=1,2, \ldots, 2 n .
$$

Because the Hamiltonian is quadratic, the differential equation [Eq. (4)] is linear. As a result, the solution of $\boldsymbol{\alpha}^{H}(t)$ can be written in the following linear form:

$$
\boldsymbol{\alpha}^{H}(t)=D(t) \boldsymbol{\alpha}+E(t)
$$

where $D(t)$ is the general solution and $E(t)$ is the particular solution of the linear ordinary differential equation [Eq. (4)] [32]. Substituting Eq. (5) into Eq. (4), we obtain

$$
\begin{aligned}
& \dot{D}(t)=-i \tau_{B} A(t) D(t), \quad D\left(t_{0}\right)=I, \\
& \dot{E}(t)=-i \tau_{B}[A(t) E(t)+B(t)], \quad E\left(t_{0}\right)=0,
\end{aligned}
$$

where the overhead dot denotes the time derivative, $\tau_{B}$ is a constant matrix given by

$$
\tau_{B}=\left(\begin{array}{cc}
0 & \mathbf{I} \\
-\mathbf{I} & 0
\end{array}\right)
$$

and $I$ (I) denotes the $2 n \times 2 n(n \times n)$ identity matrix. Then by substituting $\boldsymbol{\alpha}^{H}\left(t_{1}\right)=D\left(t_{1}\right) \boldsymbol{\alpha}+E\left(t_{1}\right)$ into $\hat{H}_{1}^{H}=$ $\frac{1}{2} \boldsymbol{\alpha}^{H T}\left(t_{1}\right) A\left(t_{1}\right) \boldsymbol{\alpha}^{H}\left(t_{1}\right)+B^{T}\left(t_{1}\right) \boldsymbol{\alpha}^{H}\left(t_{1}\right)+C\left(t_{1}\right)$, we obtain the expression of the Hamiltonian in the Heisenberg picture. Please note that the Hamiltonian $\hat{H}_{1}^{H}$ is still a quadratic polynomial of $\boldsymbol{\alpha}$.

(2) Calculate the trace of the product of several exponential operators in Eq. (2) by utilizing the Lie-group representation technique which will be elucidated in the next section.

Similarly, we can also consider a time-dependent fermionic system whose Hamiltonian can be written as

$$
\hat{H}(t)=\frac{1}{2} \boldsymbol{\beta}^{T} A(t) \boldsymbol{\beta}+B^{T}(t) \boldsymbol{\beta}+C(t) .
$$

We would like to emphasize that when there is no linear part in Eq. (7), i.e., $B(t) \equiv 0$, the calculating method for quadratic fermionic systems is almost the same as that for quadratic bosonic systems mentioned above. However, when $B(t) \not \equiv 0$, the above method fails, because the Bogoliubov transformation does not apply in this situation [27,33-35]. For this situation, we can employ a new method which is described as follows to bypass this difficulty.

We introduce an artificial Hamiltonian $\hat{\tilde{H}}(t)$ by adding a pair of identical fermionic "ghost" operators $\hat{b}_{0}$ and $\hat{b}_{0}^{\dagger}$ [35]

$$
\begin{aligned}
\hat{H}(t) & \equiv \frac{1}{2} \boldsymbol{\beta}^{T} A(t) \boldsymbol{\beta}+\left(\hat{b}_{0}^{\dagger}-\hat{b}_{0}\right) B^{T}(t) \boldsymbol{\beta}+C(t) \\
& =\frac{1}{2} \tilde{\boldsymbol{\beta}}^{T} \tilde{A}(t) \tilde{\boldsymbol{\beta}}+C(t),
\end{aligned}
$$

where $\tilde{\boldsymbol{\beta}}=\left(\hat{b}_{0}, \hat{b}_{1}, \ldots, \hat{b}_{n}, \hat{b}_{0}^{\dagger}, \hat{b}_{1}^{\dagger}, \ldots, \hat{b}_{n}^{\dagger}\right)^{T}$. The Heisenberg equation of motion for $\tilde{\boldsymbol{\beta}}^{H}(t)=\left(\hat{b}_{0}^{H}(t), \hat{b}_{1}^{H}(t), \ldots, \hat{b}_{n}^{H}(t)\right.$, $\left.\hat{b}_{0}^{\dagger H}(t), \hat{b}_{1}^{\dagger H}(t), \ldots, \hat{b}_{n}^{\dagger H}(t)\right)^{T}$ is linear again. Now, the artificial Hamiltonian has no linear part. The above method can be applied, and we obtain the CFW $\tilde{G}(v)$ corresponding to $\hat{\tilde{H}}(t)$ by similarly following the procedure for bosons. Finally, the CFW corresponding to $\hat{H}(t)$ is obtained by $G(v)=\frac{1}{2} \tilde{G}(v)$ (see Appendix A).

\section{LIE-GROUP REPRESENTATION TECHNIQUE AND TRACE FORMULA}

A key point in the calculation of the trace [Eq. (2)] is to calculate the product of several exponential operators and this can be significantly simplified by utilizing the Lie-group representation technique. A number of different representations of Lie algebras can be constructed from the bosonic (fermionic) annihilation and creation operators [36-38]. For bosons, we have ( $\hat{1}$ denotes the identity operator) 
(i) The $2 n$-dimensional Heisenberg algebra $\mathfrak{h}(n)$

$$
\hat{a}_{i}^{\dagger}, \hat{a}_{j}, \hat{1} \quad i, j=1,2, \ldots, n .
$$

(ii) The $2 n$-dimensional symplectic algebra $\mathfrak{s p}(2 n)$

$$
\hat{a}_{i}^{\dagger} \hat{a}_{j}, \hat{a}_{i}^{\dagger} \hat{a}_{j}^{\dagger}, \hat{a}_{i} \hat{a}_{j} \quad i, j=1,2, \ldots, n .
$$

(iii) The semidirect product of symplectic algebra and Heisenberg algebra $\mathfrak{s p}(2 n) \bigotimes_{s} \mathfrak{h}(n)$

$$
\hat{a}_{i}^{\dagger} \hat{a}_{j}, \hat{a}_{i}^{\dagger} \hat{a}_{j}^{\dagger}, \hat{a}_{i} \hat{a}_{j}, \hat{a}_{i}^{\dagger}, \hat{a}_{j}, \hat{1} \quad i, j=1,2, \ldots, n .
$$

And for fermions, we have

(iv) The $2 n$-dimensional special orthogonal algebra $\mathfrak{s o}(2 n)$

$$
\hat{b}_{i}^{\dagger} \hat{b}_{j}-\frac{1}{2} \delta_{i j}, \hat{b}_{i}^{\dagger} \hat{b}_{j}^{\dagger}, \hat{b}_{i} \hat{b}_{j} \quad i, j=1,2, \ldots, n,
$$

where $\delta_{i j}$ denotes the Kronecker delta function.

(v) The $2 n+1$-dimensional special orthogonal algebra $\mathfrak{s o}(2 n+1)$

$$
\hat{b}_{i}^{\dagger} \hat{b}_{j}-\frac{1}{2} \delta_{i j}, \hat{b}_{i}^{\dagger} \hat{b}_{j}^{\dagger}, \hat{b}_{i} \hat{b}_{j}, \hat{b}_{i}^{\dagger}, \hat{b}_{j}, \hat{1} \quad i, j=1,2, \ldots, n .
$$

The above algebras are all over the complex number field $\mathbb{C}$. We would like to emphasize that all quadratic Hamiltonians can be constructed by the above Lie algebras [36]. Hence, our study has exhausted [39] all situations of quantum systems described by quadratic Hamiltonians. The exponential map maps the Lie algebras to the corresponding Lie groups, the Heisenberg group $\mathrm{H}(n)$, the symplectic group $\mathrm{Sp}(2 n)$, the semidirect product of the symplectic group and Heisenberg group $\operatorname{Sp}(2 n) \bigotimes_{s} \mathrm{H}(n)$ and the spin groups $\operatorname{Spin}(2 n)$ and $\operatorname{Spin}(2 n+1)$ [36]. Thus, the product (group multiplication) of several exponential quadratic operators with or without a linear part is equal to another exponential quadratic operator with or without a linear part [38]. The trace of the latter can be obtained [40-42] by using the coherent-state representation technique for bosons and fermions. In the following, we give the trace formula corresponding to the product of several exponential quadratic operators without a linear part.

(1) Quadratic bosonic operators without a linear part [40]: $\operatorname{Sp}(2 n)$

$$
\begin{aligned}
\operatorname{Tr} & {\left[\prod_{i=1}^{m} \exp \left(\frac{1}{2} \boldsymbol{\alpha}^{T} S_{i} \boldsymbol{\alpha}\right)\right] } \\
& =\left\{(-1)^{n} \operatorname{det}\left[\prod_{i=1}^{m} \exp \left(\tau_{B} S_{i}\right)-I\right]\right\}^{-\frac{1}{2}},
\end{aligned}
$$

where $m$ denotes the number of the exponential operators.

(2) Quadratic fermionic operators without a linear part: $\operatorname{Spin}(2 n)$

$$
\operatorname{Tr}\left[\prod_{i=1}^{m} \exp \left(\frac{1}{2} \boldsymbol{\beta}^{T} R_{i} \boldsymbol{\beta}\right)\right]=\left\{\operatorname{det}\left[\prod_{i=1}^{m} \exp \left(\tau_{F} R_{i}\right)+I\right]\right\}^{\frac{1}{2}},
$$

where

$$
\tau_{F}=\left(\begin{array}{ll}
0 & \mathbf{I} \\
\mathbf{I} & 0
\end{array}\right)
$$

$S_{i}\left(R_{i}\right)$ are $(2 n \times 2 n)$ symmetric (antisymmetric) matrices, and $\exp \left(\tau_{B} S_{i}\right)\left(\exp \left(\tau_{F} R_{i}\right)\right)$ are the $2 n$-dimensional representation matrices of $\operatorname{Sp}(2 n)(\operatorname{Spin}(2 n))$, acting on the vector space spanned by the basis $\left\{\alpha_{1}, \alpha_{2}, \ldots, \alpha_{2 n}\right\}\left(\left\{\beta_{1}, \beta_{2}, \ldots, \beta_{2 n}\right\}\right)$. The symmetric (antisymmetric) condition of $S_{i}\left(R_{i}\right)$ is due to the commutation (anticommutation) relation of bosons (fermions) [38]. Also, we require that the operators in the trace are trace class operators and $\tau_{B} S_{i}\left(\tau_{F} R_{i}\right)$ are invertible and diagonalizable [26]. We would like to emphasize that similar results to Eq. (10) have been obtained in Ref. [43] by utilizing Majorana operators.

For quadratic bosonic operators with a linear part, we can also use the coherent-state representation and Weyl ordering rule [44] to obtain the trace formula:

(3) Quadratic bosonic operators with a linear part: $\operatorname{Sp}(2 n) \bigotimes_{S} \mathrm{H}(n)$

$$
\begin{aligned}
& \operatorname{Tr}\left[\exp \left(\frac{1}{2} \boldsymbol{\alpha}^{T} S_{1} \boldsymbol{\alpha}+l_{1}^{T} \boldsymbol{\alpha}\right) \exp \left(\frac{1}{2} \boldsymbol{\alpha}^{T} S_{2} \boldsymbol{\alpha}+l_{2}^{T} \boldsymbol{\alpha}\right)\right] \\
& \quad=\left\{(-1)^{n} \operatorname{det}\left[\exp \left(\tau_{B} S_{1}\right) \exp \left(\tau_{B} S_{2}\right)-I\right]\right\}^{-\frac{1}{2}} \exp (L),
\end{aligned}
$$

where $l_{1}$ and $l_{2}$ are $2 n$-dimensional column vectors. Here $L$ reads

$$
L=\sum_{i=1}^{2} \frac{1}{2} l_{i}^{T}\left[q\left(\tau_{B} S_{i}\right)-I\right] S_{i}^{-1} l_{i}-\frac{1}{2} l^{T} S^{-1} l,
$$

where

$$
\begin{aligned}
q(x) & =\frac{\operatorname{Tanh}(x)}{x}, \\
S & =S_{1} q\left(\tau_{B} S_{1}\right)+S_{2} q\left(\tau_{B} S_{2}\right), \\
l^{T} & =l_{1}^{T} q\left(\tau_{B} S_{1}\right)+l_{2}^{T} q\left(\tau_{B} S_{2}\right) .
\end{aligned}
$$

For quadratic fermionic operators with a linear part, it is a bit tricky. By adding a pair of ghost operators (see Appendix A) [35] and using Eq. (10), we obtain the trace formula

(4) Quadratic fermionic operators with a linear part: $\operatorname{Spin}(2 n+1) \subset \operatorname{Spin}(2 n+2)$

$$
\begin{aligned}
\operatorname{Tr} & {\left[\prod_{i=1}^{m} \exp \left(\frac{1}{2} \boldsymbol{\beta}^{T} R_{i} \boldsymbol{\beta}+l_{i}^{T} \boldsymbol{\beta}\right)\right] } \\
& =\frac{1}{2}\left\{\operatorname{det}\left[\prod_{i=1}^{m} \exp \left(\tau_{B} \tilde{R}_{i}\right)+I\right]\right\}^{\frac{1}{2}},
\end{aligned}
$$

where $\tilde{R}_{i}$ is determined by the equation

$$
\frac{1}{2} \tilde{\boldsymbol{\beta}}^{T} \tilde{R}_{i} \tilde{\boldsymbol{\beta}}=\frac{1}{2} \boldsymbol{\beta}^{T} R_{i} \boldsymbol{\beta}+\left(\hat{b}_{0}^{\dagger}-\hat{b}_{0}\right) l_{i}^{T} \boldsymbol{\beta} .
$$

We would like to emphasize that the sign of the square root of a complex number in Eqs. (9)-(11) and (14) is determined by two properties of the CFW: $G(0)=1$ and $G(v)$ is a smooth function of $v$. Moreover, some trace formulas corresponding to the subgroups of the Lie groups mentioned above are special cases of our results. For example, the Levitov's formula [45,46] which corresponds to unitary groups [39] can be reproduced from Eqs. (9) and (10).

\section{EXAMPLE}

To illustrate the effectiveness of our method, in the following we solve the CFW of a one-dimensional (1D) generic time-dependent harmonic oscillator which belongs to the group $\operatorname{Sp}(2 n) \bigotimes_{s} \mathrm{H}(n)$. 
Let us consider a generic nonhomogeneous second-order linear ordinary differential equation

$$
\ddot{x}(t)+a(t) \dot{x}(t)+\omega(t)^{2} x(t)+c(t)=0 .
$$

This equation describes a forced harmonic oscillator with a time-dependent frequency $\omega(t)$ and mass $m(t)$. In addition, it is subject to an external driving force $m(t) c(t)$. From the equation of motion [Eq. (16)], one can derive the Hamiltonian

$$
H(t)=\frac{p^{2}}{2 m(t)}+\frac{1}{2} m(t) \omega^{2}(t) x^{2}+m(t) c(t) x .
$$

Then the parameter $a(t)$ in Eq. (16) can be expressed as

$$
a(t)=\frac{d}{d t} \ln \frac{m(t)}{m\left(t_{0}\right)} .
$$

For simplicity, we set $c\left(t_{0}\right)=0$.

To quantize the system, we employ the canonical commutation relation $[\hat{x}, \hat{p}]=i$. Then the solutions of $\hat{x}^{H}(t)$ and $\hat{p}^{H}(t) \mathrm{read}$

$$
\begin{aligned}
& \hat{x}^{H}(t)=y_{1}(t) \hat{x}+y_{2}(t) \frac{\hat{p}}{m\left(t_{0}\right) \omega\left(t_{0}\right)}+f(t), \\
& \hat{p}^{H}(t)=m(t) \dot{\hat{x}}^{H}(t),
\end{aligned}
$$

with $y_{1}\left(t_{0}\right)=1, \dot{y_{1}}\left(t_{0}\right)=0, \quad y_{2}\left(t_{0}\right)=0, \dot{y_{2}}\left(t_{0}\right)=\omega\left(t_{0}\right)$, and $f\left(t_{0}\right)=0$, where $y_{1}(t)$ and $y_{2}(t)$ are the general solutions and $f(t)=\int_{t_{0}}^{t} d t^{\prime}\left[y_{1}(t) y_{2}\left(t^{\prime}\right)-y_{1}\left(t^{\prime}\right) y_{2}(t)\right] \frac{m\left(t^{\prime}\right) c\left(t^{\prime}\right)}{m\left(t_{0}\right) \omega\left(t_{0}\right)}$ is the particular solution of Eq. (16). In the derivation, we have used Abel's identity [32]

$$
\frac{y_{1}(t) \dot{y}_{2}(t)-y_{2}(t) \dot{y}_{1}(t)}{y_{1}\left(t_{0}\right) \dot{y}_{2}\left(t_{0}\right)-y_{2}\left(t_{0}\right) \dot{y}_{1}\left(t_{0}\right)}=e^{-\int_{t_{0}}^{t} d s a(s)}=\frac{m\left(t_{0}\right)}{m(t)} .
$$

Now let us introduce the ladder operators $\hat{a}=\sqrt{\frac{m_{0} \omega_{0}}{2}}(\hat{x}+$ $\left.\frac{i}{m_{0} \omega_{0}} \hat{p}\right), \hat{a}^{\dagger}=\sqrt{\frac{m_{0} \omega_{0}}{2}}\left(\hat{x}-\frac{i}{m_{0} \omega_{0}} \hat{p}\right)$, where $m_{0}=m\left(t_{0}\right)$ and $\omega_{0}=$ $\omega\left(t_{0}\right)$. Also we define the Fock states as $\hat{a}^{\dagger} \hat{a}|n\rangle=n|n\rangle, n=$ $0,1, \ldots$ Thus, we can rewrite the Hamiltonian into a quadratic polynomial of operators $\hat{a}$ and $\hat{a}^{\dagger}$ and obtain the CFW by the calculating method mentioned above. As for the initial state, we would like to calculate the CFW when the system is initially prepared in a microcanonical state or a canonical equilibrium state. For this purpose, we introduce an artificial density operator $\hat{\rho}(s)=e^{\ln s \hat{a}^{\dagger} \hat{a}}=\sum_{n} s^{n}|n\rangle\langle n|$ where $s$ is a parameter. Then, the $n$th Fock state (the microcanonical state) $\hat{\rho}_{n}=|n\rangle\langle n|$ and the canonical equilibrium state $\hat{\rho}_{\text {th }}=$ $e^{-\beta \hat{H}_{0}} / Z_{0}, Z_{0}=\operatorname{Tr}\left(e^{-\beta \hat{H}_{0}}\right)$ can be generated from $\hat{\rho}(s)$ by

$$
\begin{aligned}
\hat{\rho}_{n} & =\left.\frac{1}{n !} \frac{\partial^{n}}{\partial s^{n}} \hat{\rho}_{g}(s)\right|_{s=0}, \\
\hat{\rho}_{\mathrm{th}} & =\left.\left(1-e^{-\beta \omega_{0}}\right) \hat{\rho}(s)\right|_{s=e^{-\beta \omega_{0}}},
\end{aligned}
$$

where $\beta$ is the inverse temperature of the initial thermal state.

Using Eq. (11), we obtain the generating function of the CFW $G(v, s)$ when the initial state is chosen to be $\hat{\rho}(s)$

$$
G(v, s)=\frac{\exp \left\{-\frac{i v m_{1} c_{1}^{2}}{2 \omega_{1}^{2}}+\frac{i S_{+} \cot \left(v \omega_{1} / 2\right)+i\left(P-S_{+} R_{+}+S_{-} R_{-}\right) \cot \left[\left(i \ln s+v \omega_{0}\right) / 2\right]}{\cot ^{2}\left(v \omega_{1} / 2\right)-2 R_{+} \cot \left(v \omega_{1} / 2\right) \cot \left[\left(i \ln s+v \omega_{0}\right) / 2\right]+\cot ^{2}\left[\left(i \ln s+v \omega_{0}\right) / 2\right]}\right\}}{\sqrt{2 s\left[-1+\cos \left(v \omega_{1}\right) \cos \left(i \ln s+v \omega_{0}\right)+R_{+} \sin \left(v \omega_{1}\right) \sin \left(i \ln s+v \omega_{0}\right)\right]}},
$$

with

$$
\begin{aligned}
P & =\frac{2 m_{1}^{2} \dot{f}\left(t_{1}\right)}{m_{0} \omega_{0}}\left[\frac{c_{1}}{\omega_{1}^{2}}+f\left(t_{1}\right)\right]\left[y_{1}\left(t_{1}\right) \dot{y}_{1}\left(t_{1}\right)+y_{2}\left(t_{1}\right) \dot{y}_{2}\left(t_{1}\right)\right], \\
R_{ \pm} & =\frac{m_{1} \omega_{1}}{2 m_{0} \omega_{0}}\left\{\left[y_{1}^{2}\left(t_{1}\right)+y_{2}^{2}\left(t_{1}\right)\right] \pm \frac{1}{\omega_{1}^{2}}\left[\dot{y}_{1}^{2}\left(t_{1}\right)+\dot{y}_{2}^{2}\left(t_{1}\right)\right]\right\} \\
S_{ \pm} & =m_{1} \omega_{1}\left\{\left[\frac{c_{1}}{\omega_{1}^{2}}+f\left(t_{1}\right)\right]^{2} \pm\left[\frac{\dot{f}\left(t_{1}\right)}{\omega_{1}}\right]^{2}\right\}
\end{aligned}
$$

where $m_{1}=m\left(t_{1}\right), \omega_{1}=\omega\left(t_{1}\right)$, and $c_{1}=c\left(t_{1}\right)$. Thus, the CFW for the microcanonical initial state $G_{n}(v)$ and for the canonical initial state $G_{\mathrm{th}}(v)$ can be generated from $G(v, s)$ by

$$
\begin{aligned}
G_{n}(v) & =\left.\frac{1}{n !} \frac{\partial^{n}}{\partial s^{n}} G(v, s)\right|_{s=0}, \\
G_{\mathrm{th}}(v) & =\left.\left(1-e^{-\beta \omega_{0}}\right) G(v, s)\right|_{s=\mathrm{e}^{-\beta \omega_{0}}} .
\end{aligned}
$$

It is straightforward to check that the above expressions of the CFW, $G_{n}(v)$ and $G_{\mathrm{th}}(v)$, satisfy the following conditions: (1) $G_{n}(0)=G_{\mathrm{th}}(0)=1$; (2) $G_{\mathrm{th}}(i \beta)=Z_{1} / Z_{0}$, where $Z_{1}=$ $\operatorname{Tr}\left(e^{-\beta \hat{H}_{1}}\right)$. The two conditions indicate the normalization condition of the CFW and the validity of the Jarzynski equality. We would like to emphasize that our results [Eq. (23)] include the results of the forced harmonic oscillator [11] and the results of the harmonic oscillator with a time-dependent frequency [14] as special cases. That is, for a forced harmonic oscillator, $m(t)=m_{0}, \omega(t)=\omega_{0}$, we reproduce Eqs. (30) and (33) in Ref. [11] $\left(S_{+}\right.$is denoted as $|z|^{2}$ in that reference). For a harmonic oscillator with a time-dependent frequency, $m(t)=m_{0}, c(t)=0$, we reproduce Eq. (17) in Ref. [14] $\left(R_{+}\right.$ is denoted as $Q$ in that reference). As for other examples, the $\mathrm{CFW}$ associated with the dynamical Casimir effect is obtained in Ref. [47] which belongs to the group $\operatorname{Sp}(2 n)$. And the CFW of a 1D transverse XY model is given in Appendix B which belongs to the group $\operatorname{Spin}(2 n)$. To demonstrate the powerfulness of our method, we compare the results obtained from the Lie-group representation technique with previous results in Table I.

\section{CONCLUSION}

By extending the matrix representation technique introduced in Refs. [38,40], we obtain the trace formulas of products of several exponential quadratic operators based on Liegroup theory. Our results are valid as long as the Hamiltonian can be written in the form of the quadratic polynomial of bosonic or fermionic operators which is ubiquitous in physics especially in quantum many-body systems, and our trace formulas exhausted all possible Lie groups for quadratic Hamiltonians. Hence, it is the maximum extension of Levitov's formula $[45,46]$. By utilizing these trace formulas, we develop a systematic and general procedure for calculating the work 
TABLE I. Comparison of results obtained from our method with previous results.

\begin{tabular}{|c|c|c|}
\hline & Group-theoretical approach & Previous results \\
\hline \multirow{4}{*}{ Boson } & Generic time-dependent & $\begin{array}{l}\text { Harmonic oscillator with a time-dependent frequency } \\
\left.\text { [when } m(t)=m_{0}, c(t)=0\right][14,15]\end{array}$ \\
\hline & harmonic oscillator: $\operatorname{Sp}(2 n) \bigotimes_{s} \mathrm{H}(n)$ & Forced harmonic oscillator \\
\hline & [arbitrary protocol: $m(t), \omega(t), c(t)][$ Eq. (23)] & [when $\left.m(t)=m_{0}, \omega(t)=\omega_{0}\right][11-13]$ \\
\hline & & $\begin{array}{l}\text { Quenched Luttinger liquid at zero temperature [8] } \\
\text { A driving quantum scalar field [16] }\end{array}$ \\
\hline \multirow{3}{*}{ Fermion } & Transverse XY model: $\operatorname{Spin}(2 n)$ & Transverse XY model \\
\hline & (arbitrary protocol) [Eq. (B23) in Appendix B] & (sudden quench protocol) [17-19] \\
\hline & $\begin{array}{l}\text { Anisotropic XY model in a magnetic field } \\
\text { parallel to X or Y axis: } \operatorname{Spin}(2 n+1)[35,48]\end{array}$ & \\
\hline
\end{tabular}

distributions in many quantum systems under arbitrary work protocols in a much more efficient way. In comparison with previous results which are restricted to either several specific models or very special protocols, we can obtain the analytical expression of the CFW by a unified method. In addition, our method reduces the cost of the computational resource from the exponential growth (the dimension of Hilbert space) to the polynomial growth (the dimension of the representation space of the Lie group) with the number of the modes. As examples, we calculate the $\mathrm{CFW}$ of a generic time-dependent harmonic oscillator and the CFW of a time-dependent transverse XY model to illustrate the powerfulness of our method. We also would like to emphasize that this method has potential applications in many other fields, such as quantum optics and statistical physics. Applications include the partition functions, out-of-time-ordered correlation functions [49-51], Loschmidt echoes [52,53], full-counting statistics [54,55], and other correlation functions. Studies about applying our method to these problems will be given in forthcoming papers.

\section{ACKNOWLEDGMENT}

H.T.Q. gratefully acknowledges support from the National Science Foundation of China under Grants No. 11775001, No. 11534002, and No. 11825001.

\section{APPENDIX A: GHOST OPERATOR METHOD FOR FERMIONIC SYSTEM}

Let $\mathcal{H}$ denote the $2^{n}$-dimensional Hilbert space of a fermionic system with the basis vectors $\left|\left\{n_{i}\right\}\right\rangle \equiv \otimes_{i}\left|n_{i}\right\rangle, n_{i}=$ 0,1 and the ladder operators $\hat{b}_{j}, \hat{b}_{j}^{\dagger}(i, j=1,2, \ldots, n)$. We introduce a $2^{n+1}$-dimensional Hilbert space $\tilde{\mathcal{H}}$ by adding a pair of fermionic ghost operators $\hat{b}_{0}, \hat{b}_{0}^{\dagger}$ with the basis vectors $|0\rangle,|1\rangle$. These operators obey the anticommutation relation

$$
\begin{aligned}
& \left\{\hat{b}_{i}, \hat{b}_{j}^{\dagger}\right\}=\delta_{i j}, \\
& \left\{\hat{b}_{i}, \hat{b}_{j}\right\}=\left\{\hat{b}_{i}^{\dagger}, \hat{b}_{j}^{\dagger}\right\}=0, \quad i, j=0,1, \ldots, n,
\end{aligned}
$$

where $\{\hat{U}, \hat{V}\}=\hat{U} \hat{V}+\hat{V} \hat{U}$. Then, the Hilbert space $\tilde{\mathcal{H}}$ can be divided into two $2^{n}$-dimensional subspaces $\tilde{\mathcal{H}}=\tilde{\mathcal{H}}_{+} \oplus \tilde{\mathcal{H}}_{-}$ with the basis vectors $\left|\left\{n_{i}\right\}\right\rangle_{+} \oplus\left|\left\{n_{i}\right\}\right\rangle_{-}$, where

$$
\left|\left\{n_{i}\right\}\right\rangle_{ \pm}=\frac{1}{\sqrt{2}}(|0\rangle \pm|1\rangle) \otimes\left|\left\{n_{i}\right\}\right\rangle .
$$

Correspondingly, the orthogonal projective operators on the two subspaces are $\hat{P}_{ \pm}=\frac{1}{2}\left[\hat{1} \pm\left(\hat{b}_{0}+\hat{b}_{0}^{\dagger}\right)\right]$, where $\hat{1}$ denotes the identity operator. According to Ref. [35], we construct an isomorphic mapping between the Hilbert space $\mathcal{H}$ and the subspace $\tilde{\mathcal{H}}_{+}$in the following way:

$$
\begin{aligned}
\left|\left\{n_{i}\right\}\right\rangle & \leftrightarrow\left|\left\{n_{i}\right\}\right\rangle_{+}, \\
\hat{b}_{j} & \leftrightarrow\left(\hat{b}_{0}^{\dagger}-\hat{b}_{0}\right) \hat{b}_{j}, \\
\hat{b}_{j}^{\dagger} & \leftrightarrow\left(\hat{b}_{0}^{\dagger}-\hat{b}_{0}\right) \hat{b}_{j}^{\dagger}, \\
f\left(\left\{\hat{b}_{j}\right\},\left\{\hat{b}_{j}^{\dagger}\right\}\right) & \leftrightarrow f\left(\left\{\left(\hat{b}_{0}^{\dagger}-\hat{b}_{0}\right) \hat{b}_{j}\right\},\left\{\left(\hat{b}_{0}^{\dagger}-\hat{b}_{0}\right) \hat{b}_{j}^{\dagger}\right\}\right),
\end{aligned}
$$

where $i, j=1,2, \ldots, n, f\left(\left\{\hat{b}_{j}\right\},\left\{\hat{b}_{j}^{\dagger}\right\}\right) \equiv f\left(\hat{b}_{1}, \hat{b}_{2}, \ldots, \hat{b}_{n}, \hat{b}_{1}^{\dagger}\right.$, $\left.\hat{b}_{2}^{\dagger}, \ldots, \hat{b}_{n}^{\dagger}\right)$ is an arbitrary smooth function of $\hat{b}_{j}$ and $\hat{b}_{j}^{\dagger}$. Hence, if $f\left(\left\{\hat{b}_{j}\right\},\left\{\hat{b}_{j}^{\dagger}\right\}\right)$ is trace class, we can calculate $\operatorname{Tr}\left[f\left(\left\{\hat{b}_{j}\right\},\left\{\hat{b}_{j}^{\dagger}\right\}\right)\right]$ both in $\mathcal{H}$ and in $\tilde{\mathcal{H}}$ :

$$
\begin{aligned}
\operatorname{Tr}[ & \left.f\left(\left\{\hat{b}_{j}\right\},\left\{\hat{b}_{j}^{\dagger}\right\}\right)\right]=\sum_{\left\{n_{i}\right\}}\left\langle\left\{n_{i}\right\}\left|f\left(\left\{\hat{b}_{j}\right\},\left\{\hat{b}_{j}^{\dagger}\right\}\right)\right|\left\{n_{i}\right\}\right\rangle \\
= & \sum_{\left\{n_{i}\right\}}+\left\langle\left\{n_{i}\right\}\left|f\left(\left\{\left(\hat{b}_{0}^{\dagger}-\hat{b}_{0}\right) \hat{b}_{j}\right\},\left\{\left(\hat{b}_{0}^{\dagger}-\hat{b}_{0}\right) \hat{b}_{j}^{\dagger}\right\}\right)\right|\left\{n_{i}\right\}\right\rangle_{+} \\
= & \sum_{n_{0},\left\{n_{i}\right\}}\left\langle n_{0}\right| \otimes\left\langle\left\{n_{i}\right\}\right| \hat{P}_{+} f\left(\left\{\left(\hat{b}_{0}^{\dagger}-\hat{b}_{0}\right) \hat{b}_{j}\right\},\right. \\
& \left.\left\{\left(\hat{b}_{0}^{\dagger}-\hat{b}_{0}\right) \hat{b}_{j}^{\dagger}\right\}\right) \hat{P}_{+}\left|n_{0}\right\rangle \otimes\left|\left\{n_{i}\right\}\right\rangle \\
= & \operatorname{Tr}\left[\hat{P}_{+} f\left(\left\{\left(\hat{b}_{0}^{\dagger}-\hat{b}_{0}\right) \hat{b}_{j}\right\},\left\{\left(\hat{b}_{0}^{\dagger}-\hat{b}_{0}\right) \hat{b}_{j}^{\dagger}\right\}\right) \hat{P}_{+}\right] \\
= & \operatorname{Tr}\left[\hat{P}_{+} f\left(\left\{\left(\hat{b}_{0}^{\dagger}-\hat{b}_{0}\right) \hat{b}_{j}\right\},\left\{\left(\hat{b}_{0}^{\dagger}-\hat{b}_{0}\right) \hat{b}_{j}^{\dagger}\right\}\right)\right],
\end{aligned}
$$

where we have used the property $\hat{P}_{ \pm}^{2}=\hat{P}_{ \pm}$. Finally, because $f\left(\left\{\left(\hat{b}_{0}^{\dagger}-\hat{b}_{0}\right) \hat{b}_{j}\right\},\left\{\left(\hat{b}_{0}^{\dagger}-\hat{b}_{0}\right) \hat{b}_{j}^{\dagger}\right\}\right)$ commutes with the parity operator $\hat{\Pi}=\exp \left(i \pi \sum_{i=0}^{n} \hat{b}_{i}^{\dagger} \hat{b}_{i}\right)$, we have

$$
\begin{aligned}
\operatorname{Tr} & {\left[\hat{P}_{+} f\left(\left\{\left(\hat{b}_{0}^{\dagger}-\hat{b}_{0}\right) \hat{b}_{j}\right\},\left\{\left(\hat{b}_{0}^{\dagger}-\hat{b}_{0}\right) \hat{b}_{j}^{\dagger}\right\}\right)\right] } \\
& =\operatorname{Tr}\left[\hat{\Pi}^{-1} \hat{\Pi} \hat{P}_{+} f\left(\left\{\left(\hat{b}_{0}^{\dagger}-\hat{b}_{0}\right) \hat{b}_{j}\right\},\left\{\left(\hat{b}_{0}^{\dagger}-\hat{b}_{0}\right) \hat{b}_{j}^{\dagger}\right\}\right)\right] \\
& =\operatorname{Tr}\left[\hat{\Pi} \hat{P}_{+} \hat{\Pi}^{-1} f\left(\left\{\left(\hat{b}_{0}^{\dagger}-\hat{b}_{0}\right) \hat{b}_{j}\right\},\left\{\left(\hat{b}_{0}^{\dagger}-\hat{b}_{0}\right) \hat{b}_{j}^{\dagger}\right\}\right)\right] \\
& =\operatorname{Tr}\left[\hat{P}_{-} f\left(\left\{\left(\hat{b}_{0}^{\dagger}-\hat{b}_{0}\right) \hat{b}_{j}\right\},\left\{\left(\hat{b}_{0}^{\dagger}-\hat{b}_{0}\right) \hat{b}_{j}^{\dagger}\right\}\right)\right]
\end{aligned}
$$




$$
\begin{aligned}
& =\frac{1}{2} \operatorname{Tr}\left[\left(\hat{P}_{+}+\hat{P}_{-}\right) f\left(\left\{\left(\hat{b}_{0}^{\dagger}-\hat{b}_{0}\right) \hat{b}_{j}\right\},\left\{\left(\hat{b}_{0}^{\dagger}-\hat{b}_{0}\right) \hat{b}_{j}^{\dagger}\right\}\right)\right] \\
& =\frac{1}{2} \operatorname{Tr}\left[f\left(\left\{\left(\hat{b}_{0}^{\dagger}-\hat{b}_{0}\right) \hat{b}_{j}\right\},\left\{\left(\hat{b}_{0}^{\dagger}-\hat{b}_{0}\right) \hat{b}_{j}^{\dagger}\right\}\right)\right] .
\end{aligned}
$$

\section{APPENDIX B: 1D TRANSVERSE XY MODEL}

Let us consider a 1D transverse XY model described by the following Hamiltonian [48,53,56,57]:

$\hat{H}=-\frac{J}{2} \sum_{l=1}^{N}\left[(1+\gamma) \hat{\sigma}_{l}^{x} \hat{\sigma}_{l+1}^{x}+(1-\gamma) \hat{\sigma}_{l}^{y} \hat{\sigma}_{l+1}^{y}\right]-\Gamma \sum_{l=1}^{N} \hat{\sigma}_{l}^{z}$,

where $x, y, z$ are the Cartesian coordinates, $\hat{\sigma}_{l}^{x, y, z}$ are Pauli operators, $J$ is the coupling strength, $\gamma$ is the anisotropic parameter, and $\Gamma$ denotes the strength of the external magnetic field in the $z$ direction. Also, we assume the Born-von Kármán cyclic condition and the lattice points are labeled with $l=$ $1,2, \ldots, N, N+l \equiv l$. We assume $N$ to be a even number for simplicity.

As usual, we use the Jordan-Wigner transformation $[48,56-58]$ to simplify the system,

$$
\begin{gathered}
\hat{\sigma}_{l}^{z}=2 \hat{b}_{l}^{\dagger} \hat{b}_{l}-1, \\
\hat{\sigma}_{l}^{x}=\left(\hat{b}_{l}^{\dagger}+\hat{b}_{l}\right) \prod_{m<l}\left(1-2 \hat{b}_{m}^{\dagger} \hat{b}_{m}\right), \\
\hat{\sigma}_{l}^{y}=-i\left(\hat{b}_{l}^{\dagger}-\hat{b}_{l}\right) \prod_{m<l}\left(1-2 \hat{b}_{m}^{\dagger} \hat{b}_{m}\right) .
\end{gathered}
$$

For later convenience, we define the parity operator $\hat{\Pi}=$ $\exp \left(i \pi \sum_{l=1}^{N} \hat{b}_{l}^{\dagger} \hat{b}_{l}\right)=\prod_{l=1}^{N}\left(1-2 \hat{b}_{l}^{\dagger} \hat{b}_{l}\right)$ and the corresponding projectors $\hat{P}^{ \pm}=\frac{1}{2}(1 \pm \hat{\Pi})$ on the subspaces in which the even $(+)$ or odd $(-)$ number of lattices are occupied. We would like to emphasize that the parity operator is a conserved quantity $[\hat{\Pi}, \hat{H}]=0$ even when the parameters in the Hamiltonian are time dependent. Then, we transform the XY chain into a spinless fermionic system [48,56-58]

$$
\hat{H}=\hat{P}^{+} \hat{H}^{+}+\hat{P}^{-} \hat{H}^{-},
$$

where

$$
\begin{aligned}
\hat{H}^{ \pm}= & -\Gamma \sum_{l=1}^{N}\left(\hat{b}_{l}^{\dagger} \hat{b}_{l}-\hat{b}_{l} \hat{b}_{l}^{\dagger}\right)-\gamma J \sum_{l=1}^{N}\left(\hat{b}_{l}^{\dagger} \hat{b}_{l+1}^{\dagger}-\hat{b}_{l} \hat{b}_{l+1}\right) \\
& -J \sum_{l=1}^{N}\left(\hat{b}_{l}^{\dagger} \hat{b}_{l+1}-\hat{b}_{l} \hat{b}_{l+1}^{\dagger}\right)
\end{aligned}
$$

are the reduced Hamiltonians. And $\hat{b}_{l}$ in $\hat{H}^{+}\left(\hat{H}^{-}\right)$satisfy antiperiodic boundary conditions $\hat{b}_{l+N}=-\hat{b}_{l}$ (periodic boundary conditions $\hat{b}_{l+N}=\hat{b}_{l}$ ). Furthermore, the Hamiltonian can be diagonalized by the discrete Fourier transform [58]

$$
\begin{gathered}
\hat{b}_{l}=\frac{e^{-i \pi / 4}}{\sqrt{N}} \sum_{k \in K^{ \pm}} \hat{b}_{k} e^{i k l}, \\
\hat{H}^{ \pm}=-\sum_{k \in K^{ \pm}}(\Gamma+J \cos k)\left(\hat{b}_{k}^{\dagger} \hat{b}_{k}-\hat{b}_{k} \hat{b}_{k}^{\dagger}\right) \\
-\gamma J \sum_{k \in K^{ \pm}} \sin k\left(\hat{b}_{k}^{\dagger} \hat{b}_{-k}^{\dagger}+\hat{b}_{-k} \hat{b}_{k}\right),
\end{gathered}
$$

where $K^{+}=\{\pi n / N\}, n=1-N, 3-N, \ldots, N-1$. and $K^{-}=\{\pi n / N\}, n=2-N, 4-N, \ldots, N$, and the Bogoliubov transformation [19]

$$
\hat{b}_{ \pm k}=\hat{\zeta}_{ \pm k} \cos \left(\frac{\phi_{k}}{2}\right) \pm \hat{\zeta}_{\mp k}^{\dagger} \sin \left(\frac{\phi_{k}}{2}\right)
$$

where

$$
\begin{aligned}
\cos \left(\phi_{k}\right) & =-\frac{2(\Gamma+J \cos k)}{\omega_{k}}, \\
\sin \left(\phi_{k}\right) & =\frac{2 \gamma J \sin k}{\omega_{k}}
\end{aligned}
$$

with $\omega_{k}=2 \sqrt{(\Gamma+J \cos k)^{2}+(\gamma J \sin k)^{2}}$ being the eigenenergy when $k \neq 0, \pi$. When $k=0, \pi, \phi_{k}=0$ and $w_{k}=$ $-2(\Gamma+J),-2(\Gamma-J)$. Finally, we obtain the diagonal Hamiltonian with the quasiparticle operators $\hat{\zeta}_{k}$ and $\hat{\zeta}_{k}^{\dagger}$

$$
\hat{H}^{ \pm}=\sum_{k \in K^{ \pm}} \omega_{k}\left(\hat{\zeta}_{k}^{\dagger} \hat{\zeta}_{k}-\frac{1}{2}\right)
$$

If $N$ is odd, the results are the same except that $K^{+}=\{\pi n / N\}$, $n=2-N, 4-N, \ldots, N$. and $K^{-}=\{\pi n / N\}, n=1-N$, $3-N, \ldots, N-1$.

When the parameters $J(t), \gamma(t)$, and $\Gamma(t)$ are time dependent, the vector $\xi_{k}=\left(\hat{b}_{k}, \hat{b}_{-k}, \hat{b}_{k}^{\dagger}, \hat{b}_{-k}^{\dagger}\right)$ is time independent and the vector $\eta_{k}(t)=\left(\hat{\zeta}_{k}(t), \hat{\zeta}_{-k}(t), \hat{\zeta}_{k}^{\dagger}(t), \hat{\zeta}_{-k}^{\dagger}(t)\right)$ is time dependent. Also, we almost do not show the time dependence explicitly in the following for the concision of symbols. Thus, the Hamiltonian can be rewritten with $\eta_{k}$

$$
\hat{H}^{ \pm}=\frac{1}{2} \sum_{\substack{k \in K^{ \pm} \\ k \geqslant 0}} \eta_{k}^{T} \Lambda_{k} \boldsymbol{\eta}_{k}
$$

where

$$
\Lambda_{k}=\left(\begin{array}{cccc}
0 & 0 & \omega_{k} & 0 \\
0 & 0 & 0 & \omega_{k} \\
-\omega_{k} & 0 & 0 & 0 \\
0 & -\omega_{k} & 0 & 0
\end{array}\right),
$$

when $k \neq 0, \pi$. When $k=0, \pi$,

$$
\Lambda_{k}=\left(\begin{array}{cc}
0 & \omega_{k} \\
-\omega_{k} & 0
\end{array}\right)
$$

Alternatively, the Hamiltonian can be rewritten with $\boldsymbol{\xi}_{k}$

$$
\hat{H}^{ \pm}=\frac{1}{2} \sum_{\substack{k \in K^{ \pm} \\ k \geqslant 0}} \xi_{k}^{T} \Omega_{k}^{T} \Lambda_{k} \Omega_{k} \xi
$$

where when $k \neq 0, \pi$,

$$
\Omega_{k}=\left(\begin{array}{cccc}
\cos \left(\frac{\phi_{k}}{2}\right) & 0 & 0 & -\sin \left(\frac{\phi_{k}}{2}\right) \\
0 & \cos \left(\frac{\phi_{k}}{2}\right) & \sin \left(\frac{\phi_{k}}{2}\right) & 0 \\
0 & -\sin \left(\frac{\phi_{k}}{2}\right) & \cos \left(\frac{\phi_{k}}{2}\right) & 0 \\
\sin \left(\frac{\phi_{k}}{2}\right) & 0 & 0 & \cos \left(\frac{\phi_{k}}{2}\right)
\end{array}\right)
$$

is the rotation matric between the two vectors. When $k=0, \pi$,

$$
\Omega_{k}=\left(\begin{array}{ll}
1 & 0 \\
0 & 1
\end{array}\right)
$$


Next, when $k \neq 0$, $\pi$, we solve the Heisenberg equations of motion of $\boldsymbol{\xi}_{k}^{H}(t)$

$$
\begin{aligned}
\frac{d}{d t} \xi_{k}^{H}(t) & =-i\left[\xi_{k}^{H}(t), H^{ \pm}(t)\right] \\
& =-i \tau_{B} \Omega_{k}^{T}(t) \Lambda_{k}(t) \Omega_{k}(t) \xi_{k}^{H}(t), \quad k \in K^{ \pm},
\end{aligned}
$$

by assuming the solution is $\boldsymbol{\xi}_{k}^{H}(t)=\Xi_{k}(t) \boldsymbol{\xi}_{k}$

$$
\Xi_{k}(t)=\left(\begin{array}{cccc}
x_{k}^{11}(t) & 0 & 0 & x_{k}^{12}(t) \\
0 & x_{k}^{11}(t) & -x_{k}^{12}(t) & 0 \\
0 & -x_{k}^{21}(t) & x_{k}^{22}(t) & 0 \\
x_{k}^{21}(t) & 0 & 0 & x_{k}^{22}(t)
\end{array}\right) .
$$

Then the time-dependent matrix $X_{k}=\left(x_{k}^{i j}\right)_{2 \times 2}$ satisfies

$$
\begin{aligned}
\frac{\partial}{\partial t} X_{k} & =-i \omega_{k}\left(\begin{array}{cc}
\cos \left(\phi_{k}\right) & -\sin \left(\phi_{k}\right) \\
-\sin \left(\phi_{k}\right) & -\cos \left(\phi_{k}\right)
\end{array}\right) X_{k}, \\
X_{k}\left(t_{0}\right) & =\left(\begin{array}{ll}
1 & 0 \\
0 & 1
\end{array}\right)
\end{aligned}
$$

due to Eq. (B18). Also, the Heisenberg equation of motion of $\boldsymbol{\eta}_{k}^{H}(t)=\Upsilon_{k}(t) \boldsymbol{\eta}_{k}$ is solved by

$$
\Upsilon_{k}(t)=\left(\begin{array}{cccc}
y_{k}^{11}(t) & 0 & 0 & y_{k}^{12}(t) \\
0 & y_{k}^{11}(t) & -y_{k}^{12}(t) & 0 \\
0 & -y_{k}^{21}(t) & y_{k}^{22}(t) & 0 \\
y_{k}^{21}(t) & 0 & 0 & y_{k}^{22}(t)
\end{array}\right),
$$

where

$$
\begin{aligned}
Y_{k}(t) & =\left(y_{k}^{i j}(t)\right)_{2 \times 2} \\
& =\left(\begin{array}{cc}
\cos \frac{\phi_{k}(t)}{2} & -\sin \frac{\phi_{k}(t)}{2} \\
\sin \frac{\phi_{k}(t)}{2} & \cos \frac{\phi_{k}(t)}{2}
\end{array}\right) X_{k}(t)\left(\begin{array}{cc}
\cos \frac{\phi_{k}\left(t_{0}\right)}{2} & \sin \frac{\phi_{k}\left(t_{0}\right)}{2} \\
-\sin \frac{\phi_{k}\left(t_{0}\right)}{2} & \cos \frac{\phi_{k}\left(t_{0}\right)}{2}
\end{array}\right) .
\end{aligned}
$$

Moreover, when $k=0, \pi$, we have $X_{k}(t)=Y_{k}(t)=\Xi_{k}(t)=$ $\Upsilon_{k}(t)$.

Finally, using Eq. (10) in the main text, we obtain the CFW of the transverse XY chain $G(v)$ with a canonical initial state

$$
\begin{aligned}
G(v) & =\frac{\operatorname{Tr}\left[e^{i v \hat{H}_{1}^{H}} e^{(-i v-\beta) \hat{H}_{0}}\right]}{\operatorname{Tr}\left(e^{-\beta \hat{H}_{0}}\right)} \\
& =\frac{\operatorname{Tr}\left[\hat{P}_{+} e^{i v \hat{H}_{1}^{+H}} e^{(-i v-\beta) \hat{H}_{0}^{+}}\right]+\operatorname{Tr}\left[\hat{P}_{-} e^{i v \hat{H}_{1}^{-H}} e^{(-i v-\beta) \hat{H}_{0}^{-}}\right]}{\operatorname{Tr}\left(\hat{P}_{+} e^{-\beta \hat{H}_{0}^{+}}\right)+\operatorname{Tr}\left(\hat{P}_{-} e^{-\beta \hat{H}_{0}^{-}}\right)} \\
& =g(v) / g(0),
\end{aligned}
$$

with

$$
g(v)=\prod_{k \in K^{+}} g_{k}^{+}(v)+\prod_{k \in K^{+}} g_{k}^{-}(v)+\prod_{k \in K^{-}} g_{k}^{+}(v)-\prod_{k \in K^{-}} g_{k}^{-}(v),
$$

where $\hat{H}_{0}^{ \pm}=\hat{H}^{ \pm}\left(t_{0}\right), \hat{H}_{1}^{ \pm H}=\hat{H}^{ \pm H}\left(t_{1}\right)$, and

$$
\begin{aligned}
g_{k}^{ \pm}(v)= & \left\{ \pm 1+\cos \left(v \omega_{k}^{1}\right) \cos \left[(v-i \beta) \omega_{k}^{0}\right]\right. \\
& \left.+Q_{k} \sin \left(v \omega_{k}^{1}\right) \sin \left[(v-i \beta) \omega_{k}^{0}\right]\right\}^{\frac{1}{2}},
\end{aligned}
$$

with $\omega_{k}^{0}=\omega_{k}\left(t_{0}\right), \omega_{k}^{1}=\omega_{k}\left(t_{1}\right)$, and

$$
Q_{k}=2 y_{k}^{11}\left(t_{1}\right) y_{k}^{22}\left(t_{1}\right)-1 .
$$

Also, the above expression of the CFW $G(v)$ satisfies the normalization condition of the CFW, $G(0)=1$, and the Jarzynski equality, $G(i \beta)=Z_{1} / Z_{0}$. If the number of the lattice is very large, i.e., $N \rightarrow \infty$, the energy spectral of the two subspaces will be the same [56,59]. Hence we have

$$
\lim _{N \rightarrow \infty} \frac{1}{N} \ln G(v)=\frac{1}{\pi} \int_{0}^{\pi} d k \ln \frac{g_{k}^{+}(v)}{g_{k}^{+}(0)},
$$

where we have used the property $Q_{k}=Q_{-k}$. Also, we obtain the first two moments of work

$$
\begin{aligned}
\langle w\rangle= & -\left.i \frac{\partial \ln G}{\partial v}\right|_{v=0} \\
= & \frac{N}{2 \pi} \int_{0}^{\pi} d k\left(\omega_{k}^{0}-Q_{k} \omega_{k}^{1}\right) \tanh \left(\frac{\beta \omega_{k}^{0}}{2}\right), \\
\sigma_{w}^{2}= & -\left.\frac{\partial^{2} \ln G}{\partial v^{2}}\right|_{v=0}=\frac{N}{4 \pi} \int_{0}^{\pi} d k\left[\left(\omega_{k}^{0}-Q_{k} \omega_{k}^{1}\right)^{2}\right. \\
& \left.+\left(1-Q_{k}^{2}\right)\left(\omega_{k}^{1}\right)^{2} \cosh \left(\beta \omega_{k}^{0}\right)\right] \operatorname{sech}^{2}\left(\frac{\beta \omega_{k}^{0}}{2}\right) .
\end{aligned}
$$

We would like to emphasize that from our main results in Eq. (B23), one can derive the CFW in the sudden quench limit and quantum adiabatic limit:

(1) Sudden quench limit:

$$
X_{k}\left(t_{1}\right)=\left(\begin{array}{ll}
1 & 0 \\
0 & 1
\end{array}\right)
$$

Hence, we have $Q_{k}=\cos \left[\phi_{k}\left(t_{1}\right)-\phi_{k}\left(t_{0}\right)\right]$. Then, the expression $\prod_{k \in K^{+}} g_{k}^{+}(v)$ is exactly Eq. (11) in Ref. [19] which is the CFW of the 1D transverse XY model in the sudden quench limit without considering the negative-parity Hamiltonian.

(2) Quantum adiabatic limit ( $N$ is finite):

$$
Y_{k}\left(t_{1}\right)=\left(\begin{array}{cc}
e^{-i \int_{t_{0}}^{t_{1}} d t \omega_{k}(t)} & 0 \\
0 & e^{i \int_{t_{0}}^{t_{1}} d t \omega_{k}(t)}
\end{array}\right) .
$$

Hence, we have $Q_{k}=1$, which is an indication of quantum adiabaticity [see Eq. (B25)].
[1] C. Jarzynski, Annu. Rev. Condens. Matter Phys. 2, 329-351 (2011).

[2] K. Sekimoto, Stochastic Energetics (Springer, New York, 2010).
[3] U. Seifert, Rep. Prog. Phys. 75, 126001 (2012).

[4] C. Jarzynski, H. T. Quan, and S. Rahav, Phys. Rev. X 5, 031038 (2015).

[5] K. Funo and H. T. Quan, Phys. Rev. Lett. 121, 040602 (2018). 
[6] J. Kurchan, arXiv:cond-mat/0007360.

[7] H. Tasaki, arXiv:cond-mat/0009244.

[8] B. Dóra, Á. Bácsi, and G. Zaránd, Phys. Rev. B 86, 161109(R) (2012).

[9] J. Goold, F. Plastina, A. Gambassi, and A. Silva, in Thermodynamics in the Quantum Regime: Recent Progress and Outlook, edited by F. Binder, L. A. Correa, C. Gogolin, J. Anders, and G. Adesso, Fundamental Theories of Physics Vol. 195 (Springer International Publishing, Cham, 2018), pp. 317-336.

[10] A. Russomanno, S. Sharma, A. Dutta, and G. E Santoro, J. Stat. Mech. (2015) P08030.

[11] P. Talkner, P. S. Burada, and P. Hänggi, Phys. Rev. E 78, 011115 (2008).

[12] J. D. Jaramillo, J. Deng, and J. Gong, Phys. Rev. E 96, 042119 (2017).

[13] H. K. Yadalam and U. Harbola, Phys. Rev. A 99, 063802 (2019).

[14] S. Deffner and E. Lutz, Phys. Rev. E 77, 021128 (2008).

[15] P. Smacchia and A. Silva, Phys. Rev. E 88, 042109 (2013).

[16] A. Ortega, E. McKay, Á. M. Alhambra, and E. MartínMartínez, Phys. Rev. Lett. 122, 240604 (2019).

[17] A. Silva, Phys. Rev. Lett. 101, 120603 (2008).

[18] F. A. Bayocboc, Jr. and F. N. C. Paraan, Phys. Rev. E 92, 032142 (2015).

[19] R. Dorner, J. Goold, C. Cormick, M. Paternostro, and V. Vedral, Phys. Rev. Lett. 109, 160601 (2012).

[20] J. Yi, P. Talkner, and M. Campisi, Phys. Rev. E 84, 011138 (2011).

[21] R. G. Lena, G. M. Palma, and G. De Chiara, Phys. Rev. A 93, 053618 (2016).

[22] J. J. Dong and Y. F. Yang, Phys. Rev. B 100, 035124 (2019).

[23] E. G. Arrais, D. A. Wisniacki, A. J. Roncaglia, and F. Toscano, arXiv:1907.06285.

[24] J. Yi, Y. W. Kim, and P. Talkner, Phys. Rev. E 85, 051107 (2012).

[25] P. Talkner, M. Morillo, J. Yi, and P. Hänggi, New J. Phys. 15, 095001 (2013).

[26] J. P. Blaizot and G. Ripka, Quantum Theory of Finite Systems (MIT Press, Cambridge, MA, 1986).

[27] N. N. Bogoliubov and N. N. Bogoliubov, Jr., Introduction to Quantum Statistical Mechanics (World Scientific, Singapore, 2010).

[28] T. Schmiedl, E. Dieterich, P. S. Dieterich, and U. Seifert, J. Stat. Mech. (2009) P07013.

[29] Z. Gong, S. Deffner, and H. T. Quan, Phys. Rev. E 90, 062121 (2014).

[30] P. Talkner, E. Lutz, and P. Hänggi, Phys. Rev. E 75, 050102(R) (2007).

[31] Here, $\boldsymbol{\alpha}$ and $\boldsymbol{\beta}$ are called "vector operators" (tensor operators of rank 1) in Lie groups (see the next section). For details about tensor operators, please refer to the book: N. Jeevanjee, $A n$
Introduction to Tensors and Group Theory for Physicists, 2nd ed. (Birkhäuser, Boston, 2015).

[32] G. Teschl, Ordinary Differential Equations and Dynamical Systems (American Mathematical Society, Providence, RI, 2012).

[33] J. W. Van Holten and K. Scharnhorst, J. Phys. A 38, 10245 (2005).

[34] N. N. Boguliubov and D. V. Shirkov, Quantum Fields (Benjamin/Cummmings, Reading, MA, 1983).

[35] J. H. P. Colpa, J. Phys. A: Math. Gen. 12, 469 (1979).

[36] R. Gilmore and D. H. Feng, Prog. Part. Nucl. Phys. 9, 479 (1983).

[37] W. M. Zhang and R. Gilmore, Rev. Mod. Phys. 62, 867 (1990).

[38] R. Balian and E. Brezin, II Nuovo Cimento B 64, 37 (1969).

[39] Actually, some subalgebras of the Lie algebras are not shown here, e.g., the unitary algebras (particle number is conserved in this situation) $\mathfrak{u}(n)=\left\{\hat{a}_{i}^{\dagger} \hat{a}_{j}, i, j=1,2, \ldots, n\right\} \subset \mathfrak{s p}(2 n)$ for bosons and $\mathfrak{u}(n)=\left\{\hat{b}_{i}^{\dagger} \hat{b}_{j}, i, j=1,2, \ldots, n\right\} \subset \mathfrak{s p i n}(2 n)$ for fermions. For details, please refer to Ref. [36].

[40] R. W. Munn and R. Silbey, J. Phys. A: Math. Gen. 11, 939 (1978).

[41] M. Combescure and D. Robert, Coherent States and Applications in Mathematical Physics (Springer Science, New York, 2012).

[42] J. W. Pan, Q. X. Dong, Y. D. Zhang, G. Hou, and X. B. Wang, Phys. Rev. E 56, 2553 (1997).

[43] I. Klich, J. Stat. Mech. (2014) P11006.

[44] L. S. Levitov and H. Lee, J. Math. Phys. 37, 4845 (1996).

[45] I. Klich, in Quantum Noise in Mesoscopic Physics (Springer, Dordrecht, 2003), p. 397.

[46] G. P. Agrawal and C. L. Mehta, J. Math. Phys. 18, 408 (1977).

[47] Z. Y. Fei, J. N. Zhang, R. Pan, T. Qiu, and H. T. Quan, Phys. Rev. A 99, 052508 (2019)

[48] E. Lieb, T. Schultz, and D. Mattis, Ann. Phys. 16, 407 (1961)

[49] I. García-Mata, M. Saraceno, R. A. Jalabert, A. J. Roncaglia, and D. A. Wisniacki, Phys. Rev. Lett. 121, 210601 (2018).

[50] M. Campisi and J. Goold, Phys. Rev. E 95, 062127 (2017).

[51] J. Tuziemski, arXiv:1903.05025.

[52] R. A. Jalabert and H. M. Pastawski, Phys. Rev. Lett. 86, 2490 (2001).

[53] H. T. Quan, Z. Song, X. F. Liu, P. Zanardi, and C. P. Sun, Phys. Rev. Lett. 96, 140604 (2006).

[54] P. Solinas and S. Gasparinetti, Phys. Rev. E 92, 042150 (2015).

[55] R. Pan, Z. Y. Fei, T. Qiu, J.-N. Zhang, and H. T. Quan, arXiv: 1904.05378

[56] S. Katisura, Phys. Rev. 127, 1508 (1962).

[57] S. Suzuki, J. I. Inoue, and B. K. Chakrabarti, Quantum Ising Phases and Transitions in Transverse Ising Models (Springer, New York, 2012).

[58] J. Dziarmaga, Phys. Rev. Lett. 95, 245701 (2005).

[59] M. Suzuki, Prog. Theor. Phys. 56, 1454 (1976). 